\title{
Continuous Cocrystallization of Benzoic Acid and Isonicotinamide by Mixing-Induced Supersaturation: Exploring Opportunities between Reactive and Antisolvent Crystallization Concepts
}

\author{
Vaclav Svoboda, ${ }^{\dagger}$ Pól MacFhionnghaile, ${ }^{\ddagger}$ John McGinty, ${ }^{\dagger}$ Lauren E. Connor, ${ }^{\S}$ Iain D. H. Oswald, ${ }^{\|}$ \\ and Jan Sefcik** \\ ${ }^{\dagger}$ EPSRC Doctoral Training Centre in Continuous Manufacturing and Crystallization, Department of Chemical and Process
Engineering, University of Strathclyde, James Weir Building, 75 Montrose Street, Glasgow G1 1XJ, United Kingdom \\ ${ }^{\ddagger}$ EPSRC Centre for Innovative Manufacturing in Continuous Manufacturing and Crystallization, Department of Chemical and \\ Process Engineering, University of Strathclyde, James Weir Building, 75 Montrose Street, Glasgow G1 1XJ, United Kingdom \\ ${ }^{\S}$ Collaborative International Research Programme, Strathclyde Institute of Pharmacy and Biomedical Sciences, University of \\ Strathclyde, Technology and Innovation Centre, 99 George Street, Glasgow G1 1RD, United Kingdom \\ "Strathclyde Institute of Pharmacy and Biomedical Sciences, University of Strathclyde, 161 Cathedral Street, Glasgow G4 0RE, United \\ Kingdom
}

\section{Supporting Information}

ABSTRACT: This study combines reactive and antisolvent crystallization concepts via mixing-induced supersaturation to demonstrate a wider range of options for solvent system selection in multicomponent crystallization. This approach was applied to investigate continuous crystallization of 1:1 and 2:1 cocrystals of benzoic acid and isonicotinamide. Design of Experiments was used to identify conditions where pure cocrystal phases are obtained and a continuous mixing-induced cocrystallization process was implemented to selectively produce either $1: 1$ or $2: 1$ cocrystals.

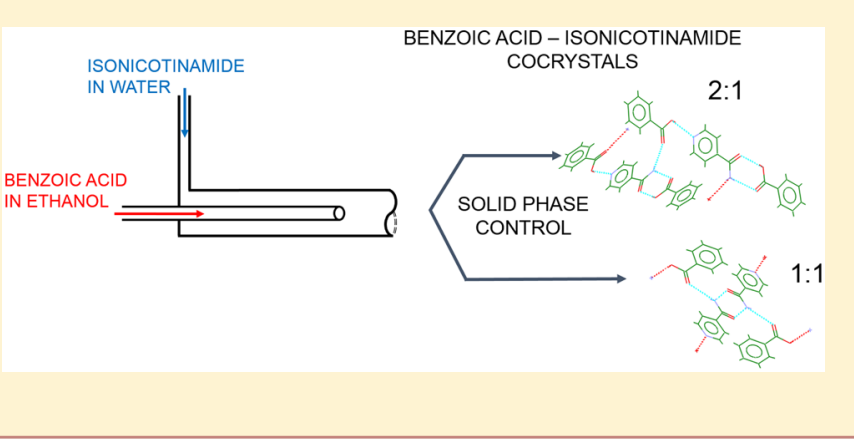

or reactive crystallization. ${ }^{11}$ Cooling cocrystallization has previously been implemented in continuous platforms ${ }^{12,13}$ and with cocrystal stoichiometry control. ${ }^{14}$ Cocrystallization using antisolvent has been previously studied in batch processes, ${ }^{10}$ but not using continuous methods. Antisolvent crystallization of single component materials has been shown adaptable to continuous manufacturing and scale up in a number of processes. ${ }^{15-17}$ Reactive cocrystallization mentioned in the work of Rodriguez-Hornedo et al. ${ }^{11}$ does not involve a chemical reaction as such but a solution-based formation of a multicomponent solid phase from a mixture of two solutions undersaturated with respect to individual components using the same solvent.

Processes that rely on mixing to induce supersaturation such as reactive and antisolvent crystallizations are well amenable to scale-up under continuous conditions. ${ }^{18}$ Continuous manufacturing of pharmaceuticals can provide benefits such as decreased plant footprint, easier scale-up, shorter lead times, and better unit performance through process intensification. ${ }^{19}$ Since mixing can have strong impact on generation of

Received: December 20, 2016

Revised: February 9, 2017

Published: February 13, 2017 
supersaturation profile and subsequent nucleation, especially under kinetically controlled conditions, ${ }^{20}$ well-controlled mixing is essential for a control over final crystal properties, such as solid form and particle size distribution.

Antisolvent and reactive crystallization approaches can be combined in order to design a process where supersaturation is induced by mixing and is determined by the position in a quaternary phase diagram involving two crystal coformers and two solvents. Depending on the shape of the phase diagram, which often shows a highly unsymmetrical nature, ${ }^{21}$ the cocrystal would be the thermodynamically most stable phase under some conditions. However, sometimes it may not be readily crystallized due to kinetic limitations (slow nucleation or growth) even if thermodynamically favored. Munshi and coworkers pointed out opportunities to use mixed solvents in controlling solid phase outcomes in cooling cocrystallization. ${ }^{22}$ Solvent selection, a key design choice in crystallization, becomes more challenging in multicomponent systems. The key parameter of cocrystallization process design is the supersaturation with respect to the cocrystal phase, rather than the supersaturations of the individual coformers. While having four components increases the complexity of mapping the phase diagram, it also allows for more options how to access solid phase regions, which might not be easily accessible at a fixed solvent composition. For example, it may be possible to start with a solution of both coformers undersaturated in one solvent and add a second solvent to induce supersaturation, as in antisolvent crystallization. Alternatively, one can start with one coformer undersaturated in a given solvent mixture, and the other coformer undersaturated in the same solvent mixture and generate supersaturation by mixing these two solutions together, as in reactive crystallization. These decisions will be driven by the shape of the phase diagram and the nature of the target solid phase.

This study combines antisolvent and reactive crystallization concepts to develop a continuous cocrystallization process to produce benzoic acid (BZA)-isonicotinamide (INA) cocrystals. These molecules form two different cocrystals in 2:1 and 1:1 stoichiometric ratios, which have been previously isolated and characterized from small scale cooling crystallization. ${ }^{23,24}$ In this work, benchtop screening crystallization experiments were scaled to run in a continuous process. A Design of Experiment was carried out for the benchtop screening to better understand the effects of different process conditions on the crystallization of the two cocrystal phases. DoE screening was done in order to cut down the time and number of experiments to find suitable continuous crystallization conditions to selectively produce either $1: 1$ or $2: 1$ cocrystals by continuous crystallization.

\section{EXPERIMENTAL SECTION}

Materials. Benzoic acid ( $\geq 99.5 \%)$, isonicotinamide (99\%), and ethanol ( $\geq 99.8 \%)$ were supplied by Sigma-Aldrich (Gillingham, UK). Deionized water was produced using the in-house Millipore Milli-Q system.

Batch Screening. Two sets of screening experiments were carried out. The first was an initial screening and the second was a systematic Design of Experiments (DoE) approach used for mapping of a limited region of the quaternary phase diagram (BZA, INA, water, ethanol) in order to identify suitable operating conditions for continuous crystallization. For both sets of screening experiments, an aqueous solution of isonicotinamide was added to an ethanolic solution of benzoic acid in a $20 \mathrm{~mL}$ vial, illustrated in Figure 1. Solutions were mixed at various ratios to obtain the total of $10 \mathrm{~g}$ of solution and

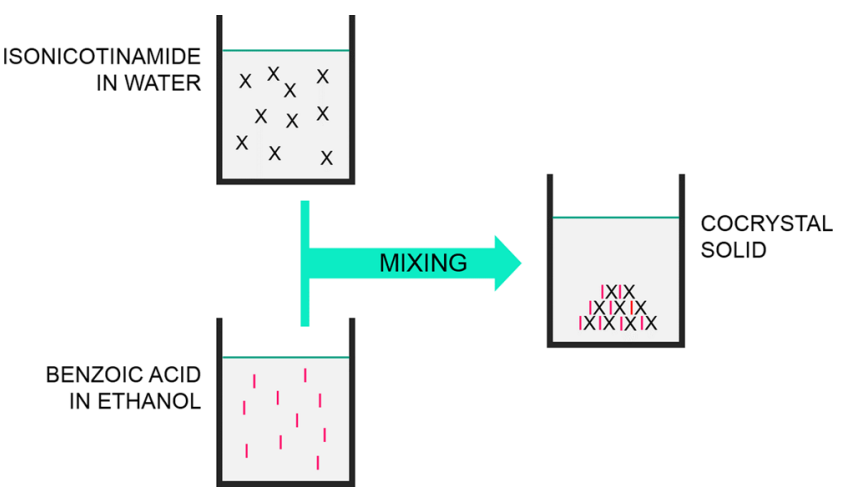

Figure 1. Solvent system used for mixing-induced cocrystallization.

agitated using magnetic stirrer bar for $10 \mathrm{~min}$ past observed nucleation. All experiments were carried out at $25{ }^{\circ} \mathrm{C}$. For the initial screen, solutions of isonicotinamide in water and benzoic acid in ethanol were prepared at concentrations of $68.1 \mathrm{~g} / \mathrm{kg}$ water and $213 \mathrm{~g} / \mathrm{kg}$ ethanol, respectively, and mixed in ratios of $2: 1,1: 1,1: 2,1: 4$, and $1: 9$ by mass. The solid product obtained was filtered using $0.2 \mu \mathrm{m}$ PTFE filter without washing, dried at $40{ }^{\circ} \mathrm{C}$ for $24 \mathrm{~h}$, and analyzed by X-ray powder diffraction (see solid characterization section). The design space for DoE was selected based on information from the initial screen. Experimental plan was created using MODDE in a 2 level full factorial design. The experimental worksheet, model fitting, and results analysis were carried out in MODDE by Umetrics. Data were fitted using partial least squares (PLS). The three variable parameters chosen for the DoE were: BZA-ethanol solution concentration, INA-water solution concentration, and the mass ratio of the two solutions mixed. Values of these three parameters combined determine the final composition of the mixture and thus the position on the phase diagram. The responses measured were induction time, solid yield, solid phases present, and resulting slurry flow properties. Induction time was estimated as time after mixing until first particles were visually observed. Solid yield was taken as a percentage of total solute that was recovered as solids by filtration $10 \mathrm{~min}$ after the estimated induction time while agitating. Slurry flow was assessed by a rating system where a number was assigned on a scale from 1 to $5: 1$ for an easily flowing mixture and 5 for a slurry too thick to flow with gravity. Solid phase was determined by XRPD (see solid characterization section). Based on this DoE, conditions for the production of 2:1 cocrystal were selected. Although the initial DoE was aimed to find suitable conditions for crystallization of both cocrystal forms, conditions leading to crystallization of 1:1 cocrystal resulted in solid loadings, which would be too high for a continuous operation. Based on results from the initial DoE, a set of further experiments was carried out in an expanded design space to find suitable conditions for crystallization of 2:1 cocrystal. These experiments were carried out at lower solution concentrations, and resulting solid phases, induction times, and slurry flow ratings were determined in the same way as in the initial DoE runs.

Solubility Measurement. Solubility of the $2: 1$ cocrystal has been determined by a solvent addition method in the Crystalline Reactor System (Technobis), similar to a previously published procedure. ${ }^{25}$ As compared to temperature variation or gravimetric methods, solvent addition relies on slow dilution of a suspension with a solvent (mixture) of a given composition under isothermal conditions until complete dissolution occurs, when a clear point can be detected. Solvent was added at a constant rate using PHD Ultra syringe pumps (Harvard Apparatus) to the crystalline vials. The solubility measurement setup is shown in Figure 2. Multiple addition rates were tested $(0.5$ and $0.75 \mathrm{~mL} / \mathrm{h})$ to verify that dissolution kinetics have a negligible effect on the result at the addition rates used. The clear point was determined using both transmissivity measurement and images from the crystalline camera. Image analysis produced more consistent results, which is in line with previous findings from Reus et al. ${ }^{25}$ A study was also carried out to monitor the solid state 


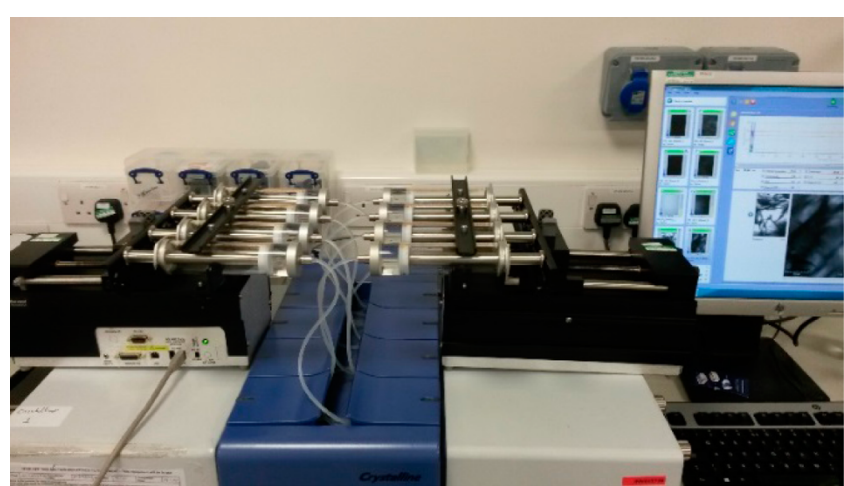

Figure 2. Technobis Crystalline and syringe pumps for solubility determination using the solvent addition method.

transformation of the cocrystal in order to check phase stability in the same setup. Suspensions with concentrations as used for the start of solubility measurements were held for varying amount of time from 1 to $5 \mathrm{~h}$, after which the remaining solid was filtered, dried, and analyzed by XRPD.

Continuous Crystallization. Continuous crystallization runs were performed using a concentric capillary mixer (Figure 3) as well as the

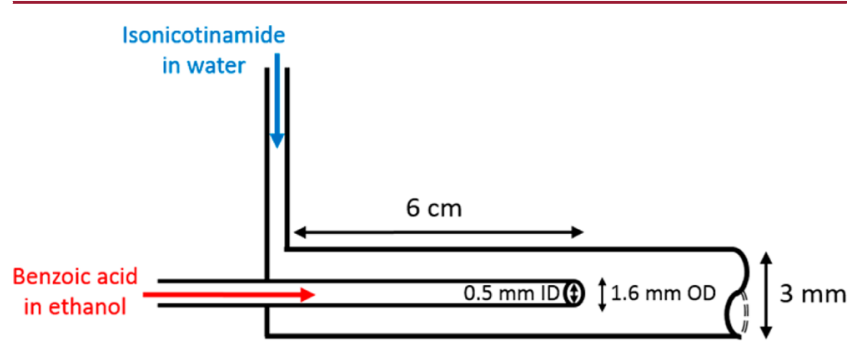

Figure 3. Concentric capillary mixer with dimensions indicated.

Ehrfeld modular microreactor system equipped with Valve Mixer 30 module (Figure 4). The BZA-ethanol solution was supplied to the

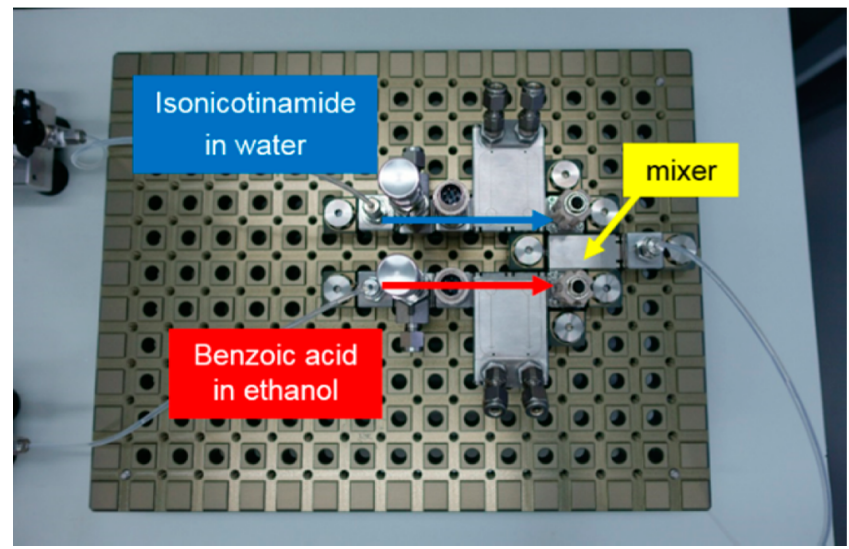

Figure 4. Ehrfeld modular microreaction system fitted with valve assisted mixer 30 .

concentric capillary mixer through the inner PEEK capillary, while the INA-water solution was fed to the outer glass tube as shown in Figure 3. The capillary has internal and outer diameters of 0.5 and $1.6 \mathrm{~mm}$, respectively. The outer glass tube has an internal diameter of $3 \mathrm{~mm}$. The capillary stream entered into the outer tube stream $6 \mathrm{~cm}$ from the $\mathrm{T}$-junction. The feed solutions were pumped using Bronkhorst Mini CORI-FLOW system coupled with gear pumps allowing for accurate control of mass flow rates. The resulting slurry from the mixer was sampled $0.7 \mathrm{~m}$ from the mixing point at the start and end of experiments. Collected slurry was filtered after a holding period in a Buchner funnel with a $0.45 \mu \mathrm{m}$ filter paper and dried overnight at 40 ${ }^{\circ} \mathrm{C}$. The slurry holding time without agitation was $10 \mathrm{~min}$ for 2:1 cocrystal run and $18 \mathrm{~min}$ for 1:1 cocrystal run. A camera was used for visual analysis of any fouling in the glass tube. Temperature of both streams was measured throughout experiments, and it varied less than $2{ }^{\circ} \mathrm{C}$ from $25{ }^{\circ} \mathrm{C}$. The total mass flow rates through the capillary mixer were 20,40 , and $60 \mathrm{~g} / \mathrm{min}$ in $50: 50(\mathrm{w} / \mathrm{w})$ ratio for producing the $2: 1$ cocrystal and $115 \mathrm{~g} / \mathrm{min}$ at a 15:100 (w/w) ratio of benzoic acid solution flow rate to isonicotinamide solution flow rate for the $1: 1$ cocrystal. In order to demonstrate the transferability of the process to a commercial platform, the Ehrfeld modular microreaction system, shown in Figure 4, was used at the total mass flow rate of $20 \mathrm{~g} / \mathrm{min}$ for producing the 2:1 cocrystal.

Solid Characterization. Filtered and dried crystalline powder from the screening and continuous experiments was analyzed by X-ray powder diffraction (XRPD) and infrared (IR) spectroscopy. Samples from continuous runs were further analyzed by differential scanning calorimetry (DSC) paired with thermogravimetric analysis (TGA) and microscope image analysis for particle size measurement. Solids from continuous runs targeted to produce the 2:1 cocrystal were also analyzed by NMR. XRPD fingerprinting was performed on a sample placed in a 28-well plate, supported by Kapton film $(7.5 \mu \mathrm{m}$ thickness). Data were collected on a Bruker AXS D8 Advance transmission diffractometer equipped with $\theta / \theta$ geometry, primary monochromatic radiation $\left(\mathrm{Cu} \mathrm{K} \alpha_{1} \lambda=1.54056 \AA\right)$, a Braun 1D position sensitive detector, and an automated multiposition $x-y$ sample stage. Data were collected from 4 to $35^{\circ} 2 \theta$ with a $0.015^{\circ} 2 \theta$ step size and $1 \mathrm{~s}$ step $^{-1}$ count time. FT-IR measurements were taken using Bruker Tensor II, using 32 scans with $4 \mathrm{~cm}^{-1}$ resolution from 450 to $4000 \mathrm{~cm}^{-1}$ with diamond tip ATR sampling plate. DSC/TGA data was obtained from Netszch STA 449 F1 Jupiter. Measurements for DSC/TGA were taken from 20 to $180{ }^{\circ} \mathrm{C}$ with a ramp of $10{ }^{\circ} \mathrm{C} /$ min. For particle size distribution (PSD), image analysis was carried out on dry powder using Malvern Morphologi G3 using low pressure dispersion and $2.5 \times$ optics. ${ }^{1} \mathrm{H}$ and ${ }^{13} \mathrm{C}$ NMR analysis was carried out using Bruker Advance 3 at $400 \mathrm{MHz}$ by dissolving the solid product in deuterated DMSO in $5 \mathrm{~mm}$ NMR vials.

\section{RESULTS AND DISCUSSION}

Mixing-Induced Supersaturation. A case study on benzoic acid-isonicotinamide cocrystallization presented here explores the space between reactive and antisolvent crystallization concepts in generating mixing-induced supersaturation to target specific solid phases of cocrystals. The illustrative phase diagram in Figure 5 is similar to the phase diagrams presented previously for the system investigated here. ${ }^{23}$ We use it to demonstrate different modes of inducing supersaturation through mixing. The blue lines illustrate examples of reactive and antisolvent crystallization. In reactive crystallization, two undersaturated solutions, each containing a single coformer in the same solvent mixture, are mixed to supersaturate with respect to a desired cocrystal solid. In antisolvent crystallization, an undersaturated solution containing both coformers in one solvent is mixed with antisolvent to generate supersaturation. The red line illustrates a new approach used in this study, where antisolvent and reactive crystallization concepts were combined to target a specific region of the phase diagram, in this case 2:1 and 1:1 cocrystal regions. In this combined approach, two undersaturated solutions, each containing a single coformer in a single (pure) solvent, are mixed to generate supersaturated solution with respect to desired cocrystal phase in the mixed solvent.

Screening. An initial screen was used to determine design space boundaries and was followed by a detailed DoE driven screen to map a design space corresponding to a region of 


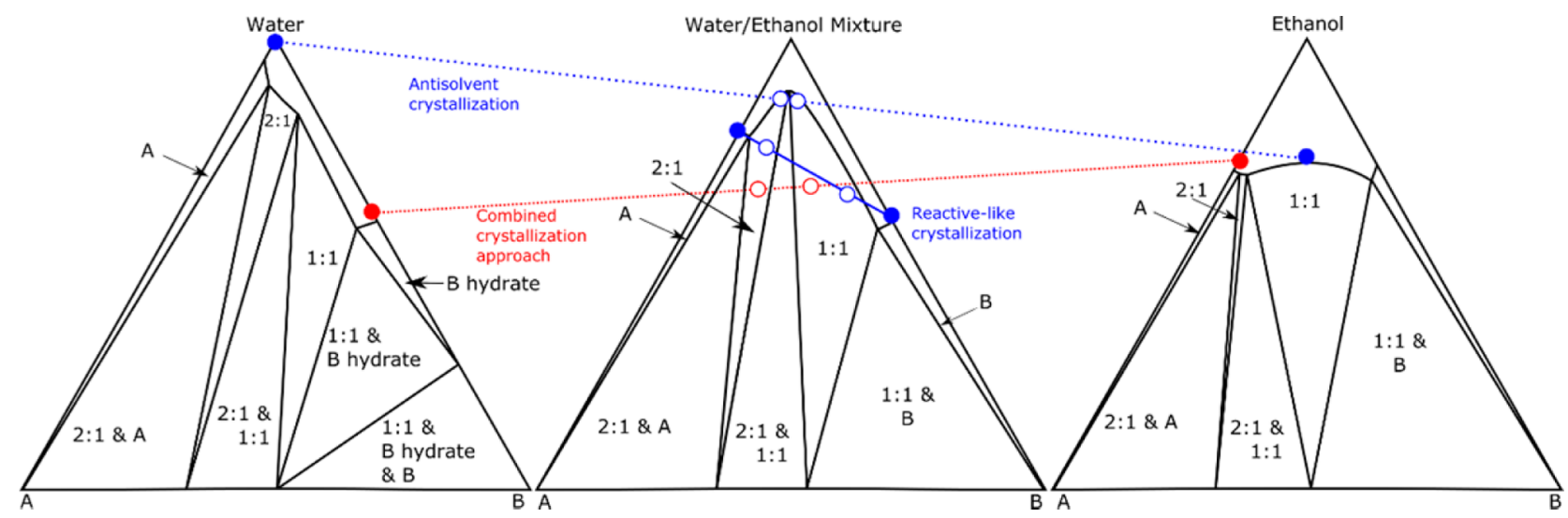

Figure 5. Illustration of three modes of inducing supersaturation through mixing: reactive, antisolvent, and combined approach. Targeted solid phases are 1:1 and 2:1 cocrystals of A and B, and solvent is a mixture of water and ethanol. Dotted lines do not correspond to lever rule.
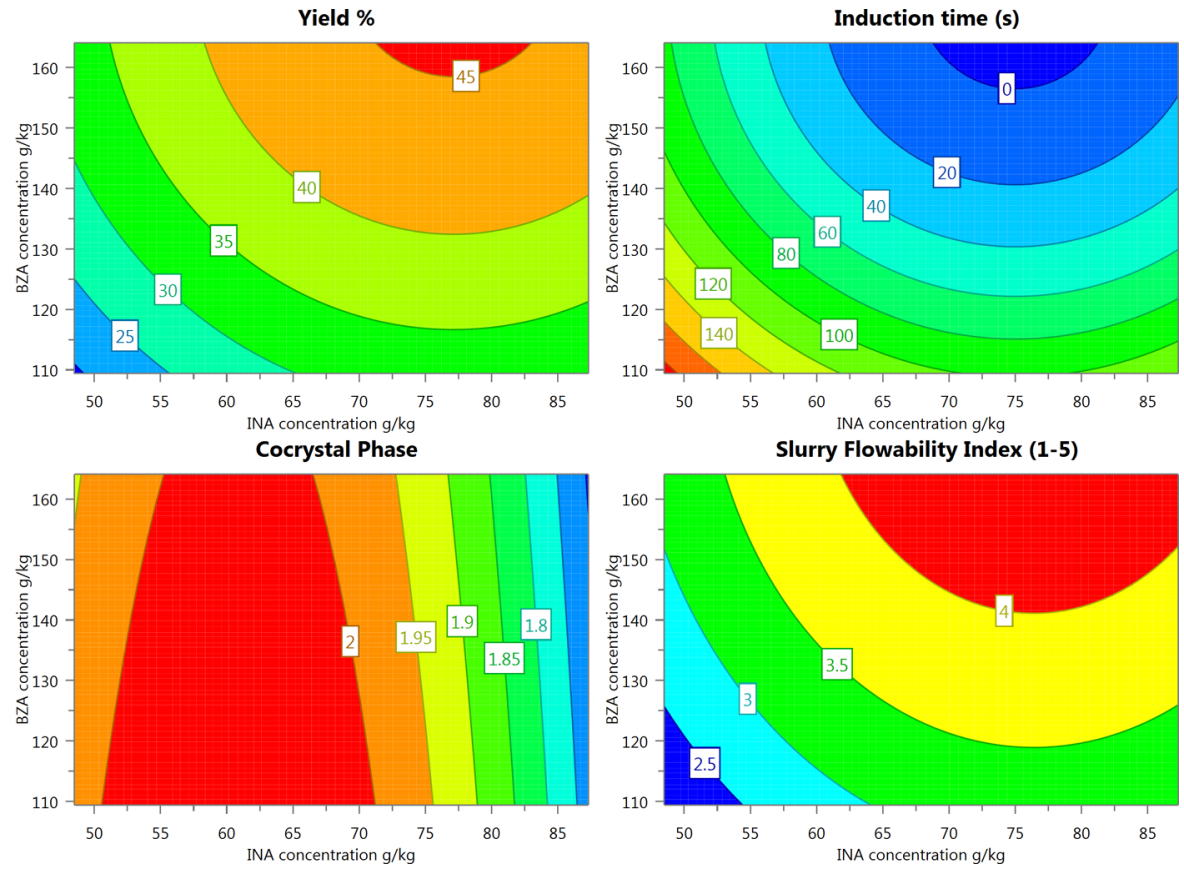

Figure 6. Contour plot showing responses from the Design of Experiments at a fixed solution ratio of 50:50 (w/w).

interest in the phase diagram. XRPD from the initial screen has shown that the method of mixing solutions of isonicotinamide in water and benzoic acid in ethanol can produce both 2:1 and 1:1 cocrystals. DoE responses were chosen as key outcomes relevant for development of a continuous crystallization process. High solid loadings at the end of experiments limited solution concentrations from above, as too high solid loadings could lead to downstream blockages. However, long induction times limited solution concentrations from below, as relatively rapid nucleation is desirable. Due to the probabilistic nature of primary nucleation, induction time estimates from batch experiments are only intended to give an approximate guidance; nevertheless, there was a good consistency among repeated experiments.

Despite not necessarily providing direct information on phase stability under thermodynamic equilibrium conditions, the DoE mapping provided a rapid way to find potentially suitable crystallization conditions while also giving indication about kinetics. Contour plots displayed in Figure 6 show responses at a fixed solution ratio where at higher concentrations of starting solutions the yield is higher, slurry flow rating diminishes due to higher solid loading and induction time decreases due to higher supersaturation. The cocrystal phase formed is primarily dependent on the solution flow ratio rather than the solution concentrations within the design space investigated. Contour plots for other solution ratios are located in Supporting Information (SI Figures 3-5). After targeting the desired phase by adjusting the solution flow ratio, slurry flow ratings of 2-3 were deemed as acceptable for a continuous crystallizer. The induction time model prediction is the least reliable as can be expected for a probabilistic process. The design space selected was large enough to find suitable conditions for the crystallization of the 2:1 cocrystal, while the conditions to crystallize the 1:1 cocrystal phase had to be extrapolated and subsequently validated. The extrapolation was done by choosing various concentrations of initial solutions outside of the original design space and analyzing the same response parameters. The conditions from the DoE chosen as most suitable to produce the 2:1 cocrystal are $50 / 50 \mathrm{w} / \mathrm{w}$ solution flow ratio, with the solution concentrations of $109.4 \mathrm{~g}$ 
$\mathrm{BZA} / \mathrm{kg}$ ethanol and $48.5 \mathrm{~g}$ INA/ $\mathrm{kg}$ water. For the $1: 1$ cocrystal the optimal conditions were the solution flow ratio of $13 / 84 \mathrm{w} /$ $\mathrm{w}$ (benzoic acid solution to isonicotinamide solution) with the solution concentrations of $30 \mathrm{~g} \mathrm{BZA} / \mathrm{kg}$ ethanol and $40 \mathrm{~g}$ INA/ $\mathrm{kg}$ water.

Solubility. During the phase stability test, the $2: 1$ cocrystal began to transform to the $1: 1$ cocrystal over time at the crystallizer outlet conditions. Transformation commenced between 158 to $264 \mathrm{~min}$ in an isothermal stirred environment as detected by XRPD, see Figure 7 . This makes solubility

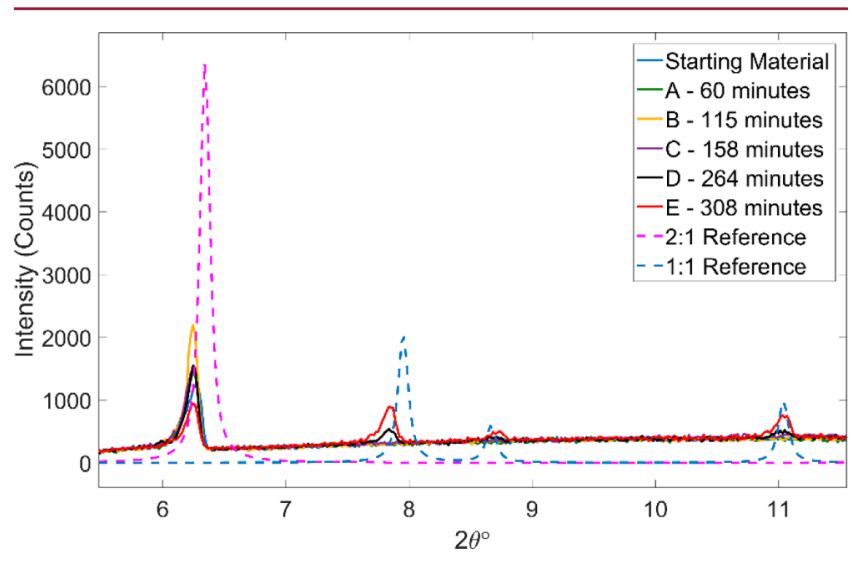

Figure 7. XRPD from suspension stability study of 2:1 cocrystal in $48.8 \%$ ethanol-water $(\mathrm{w} / \mathrm{w})$.

determination more difficult as the $2: 1$ solid form is not thermodynamically stable at the chosen process conditions. However, the solid-liquid equilibrium of the metastable solid phase was determined for the purpose of thermodynamic yield calculations. The solubility test was timed to avoid any significant transformation. Solubility measurements were carried out in $48.8 \%(\mathrm{w} / \mathrm{w})$ ethanol/water mixture, which is the same as the solvent mixture at the continuous mixer outlet during crystallization of 2:1 cocrystal. The results from six experiments suggest the solubility to be $0.0446 \mathrm{~g} / \mathrm{mL}$ solvent with a high level of reproducibility based on clear points from image analysis. The spread of various measurements is illustrated in Figure 8. The high spread of clear point measurements based on transmission may be attributed to the limited ability to detect solids by transmission measurements at low concentrations, especially for thin needles. The different solvent addition rates show comparable results; thus, the result is not dependent on the kinetics of crystal dissolution. The findings about the $2: 1$ cocrystal metastability point out that

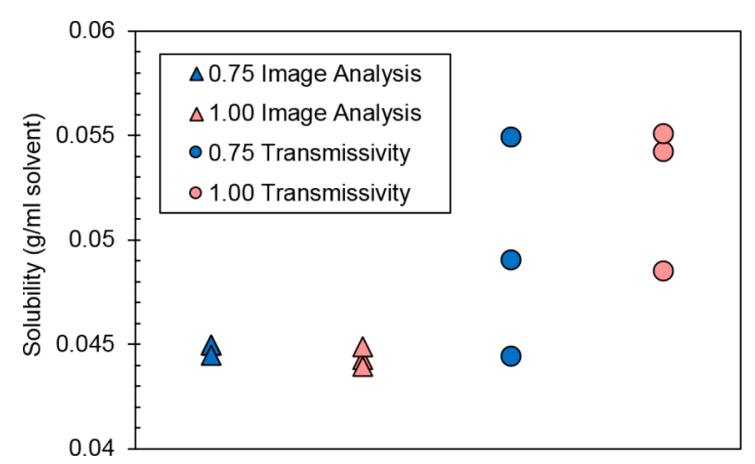

Figure 8. Solubility of 2:1 cocrystal in $48.8 \%$ ethanol-water $(w / w)$ at $25{ }^{\circ} \mathrm{C}$. the outlet composition of the crystallizer slurry is either in the $1: 1$ cocrystal or a mixture of the $1: 1$ and $2: 1$ cocrystal regions on the phase diagram. The solubility of the $2: 1$ cocrystal has been previously reported between 0.0367 and $0.0482 \mathrm{~g} / \mathrm{mL}$ for $50 \%$ water/ethanol mixture by ${ }^{26}$ using two methods. However, the measurements reported therein have a high variability, and the gravimetry method implemented did not seem to account for a possibility of a phase transformation. Therefore, it is possible that in some cases the solubility of the $1: 1$ cocrystal or a mixture of both cocrystal phases could have been measured. A recent study by Munshi et al. ${ }^{22}$ shows the ternary phase diagram at $50 \%$ ethanol/water, although it was not reported whether this was $50 \%$ by mass or by volume. ${ }^{22}$ As we have shown, solvent mediated transformation may occur in this system under conditions studied in this and previous works, and this may perhaps also help to explain the unusual shape of the phase diagram reported in the work of Munshi et al.

Continuous Crystallization. Moving from benchtop vialbased screening to a continuous platform introduces new process parameters and challenges. The overall flow rate was found to have a strong impact on the mixing regime and thus final particle properties. Three different overall flow rates were examined with the aim of producing the $2: 1$ cocrystal: 60,40 , and $20 \mathrm{~g} / \mathrm{min}$ at 50:50 ratio $(\mathrm{w} / \mathrm{w})$ of solution streams. The continuous runs revealed fouling and subsequent blockages to be a challenge; however, blockage issues decreased with lower flow rates. Antifouling measures such as low power sonication could be used to mitigate this issue. ${ }^{27}$ The solid output was $0.507,0.342$, and $0.0259 \mathrm{~g} / \mathrm{min}$ for overall flow rates of 60,40 , and $20 \mathrm{~g} / \mathrm{min}$, respectively. The $\mathrm{L} 50$ of particles obtained at overall flow rates of 60,40 , and $20 \mathrm{~g} / \mathrm{min}$ was 361,317 , and 238 $\mu \mathrm{m}$, respectively. Despite producing the desired solid form (Figure 10) from all conditions, mixing performance drastically reduced at the overall flow rate of $20 \mathrm{~g} / \mathrm{min}$ resulting in a lower solid yield. The low velocity difference between the capillary jet and the outer flow did not provide sufficient mixing to rapidly achieve the desired supersaturation. The same process at the overall flow rate of $20 \mathrm{~g} / \mathrm{min}$ resulted in $0.0484 \mathrm{~g} / \mathrm{min}$ solid output in the Ehrfeld platform. Clearly, mixing conditions influence nucleation kinetics of cocrystal as the solid yield and resulting particle size distribution (see SI Figure 6) varies with overall flow rate although the overall solution composition is the same in all cases. In order to achieve a higher yield in the crystallization process, increasing of residence time downstream from the mixing point combined with additional cooling stages should be considered. Further process optimization would benefit from more detailed knowledge of the relevant phase diagram and additional experiments in an expanded design space.

A continuous crystallization was also carried out to produce the $1: 1$ cocrystal, using flow rates of $15 \mathrm{~g} / \mathrm{min}$ of benzoic acid solution through the capillary and $100 \mathrm{~g} / \mathrm{min}$ of isonicotinamide solution through the outer tube. For this experiment the solutions were less concentrated to avoid blocking due to potentially high solid loading of the resulting slurry. The induction time at these concentrations was relatively long compared to the 2:1 cocrystal experiments and particles were not immediately visible in the mixer outlet. The solid output after holding the slurry for $18 \mathrm{~min}$ was $0.0726 \mathrm{~g} / \mathrm{min}$.

Solid Characterization. Solid state characterization techniques were selected in order to establish solid phase identity and purity. Crystal unit cell parameters were previously documented for both cocrystal phases, which makes powder X- 

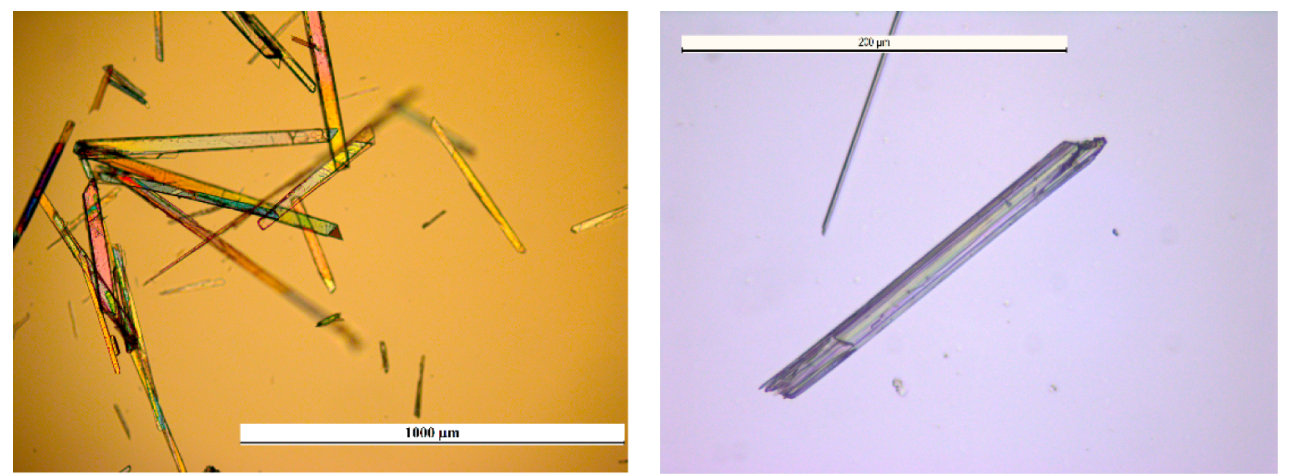

Figure 9. Optical microscope images: 2:1 cocrystal (left) and 1:1 cocrystal (right).

ray diffraction suitable for phase verification. The cocrystal phases also have unique melting points verifiable by DSC. NMR was also used to independently verify the stoichiometry of coformers in the crystalline sample. The morphological differences in crystals for the 2:1 and 1:1 forms were minor. The needle shaped morphology of both cocrystal forms can be seen in Figure 9. Results from Malvern Morphologi G3 are shown in Supporting Information, and similar aspect ratio distributions were found for both cocrystal forms. Analysis by $\mathrm{XRPD}$ on the dry samples from the capillary mixer is shown in Figure 10 (XRPD on samples from the Ehrfeld platform are in

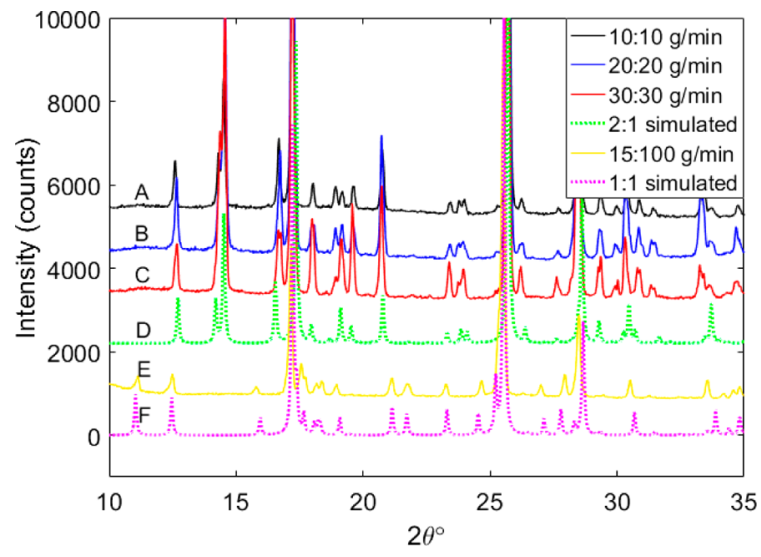

Figure 10. XRPD for samples from all continuous runs together with calculated patterns.

SI Figure 9). Lines A, B, and C are samples from continuous runs designed to produce the 2:1 cocrystal. Simulated patterns are generated from unit cell data available from the Cambridge Structural Database under structure labels: BUDWEC ${ }^{28}$ and MOVTOH $^{23}$ for the $1: 1$ and 2:1 cocrystals, respectively. Comparing with $\mathrm{D}$, the simulated pattern suggests that a pure 2:1 cocrystal phase has been produced by all runs. Similarly, line $\mathrm{E}$ shows pure 1:1 cocrystal by comparison to the $1: 1$ cocrystal simulated pattern, line F. This is further supported by results from DSC shown in Figure 11. DSC for the 2:1 cocrystal samples shows a sharp thermal event previously reported at $142-143{ }^{\circ} \mathrm{C}$ for the $2: 1$ cocrystal. ${ }^{29}$ The TGA curves also show a decrease in mass from 148 to $150{ }^{\circ} \mathrm{C}$ onward indicating sample decomposition. Similarly, DSC for 1:1 sample shows a unique endotherm above $160{ }^{\circ} \mathrm{C}$, at the expected melting point of the 1:1 cocrystal $^{29}$ with subsequent decomposition.

For additional confirmation, the samples were analyzed using ATR-IR. The absorbance spectra are in Figure 12. The figures show a spectrum for each starting material as well as samples from continuous experiments. It is clear that the cocrystal phases have a well-defined unique spectra, and it is possible to distinguish the 2:1 and 1:1 cocrystals using IR spectrometry.

NMR analyses on solid samples dissolved in deuterated DMSO from each continuous run confirmed (within 2\% error) a 2:1 ratio for each sample from the continuous runs aimed to produce 2:1 cocrystal (SI Figure 8). Continuous experiments using 40 and $60 \mathrm{~g} / \mathrm{min}$ flow rates produced particles of very similar particle size and shape characteristics. Results from 20 $\mathrm{g} / \mathrm{min}$ continuous experiment show significantly smaller particles at much lower solid yield (SI Figure 6). The aspect ratios for the particles from $20 \mathrm{~g} / \mathrm{min}$ run are more needle-like with a narrower distribution.
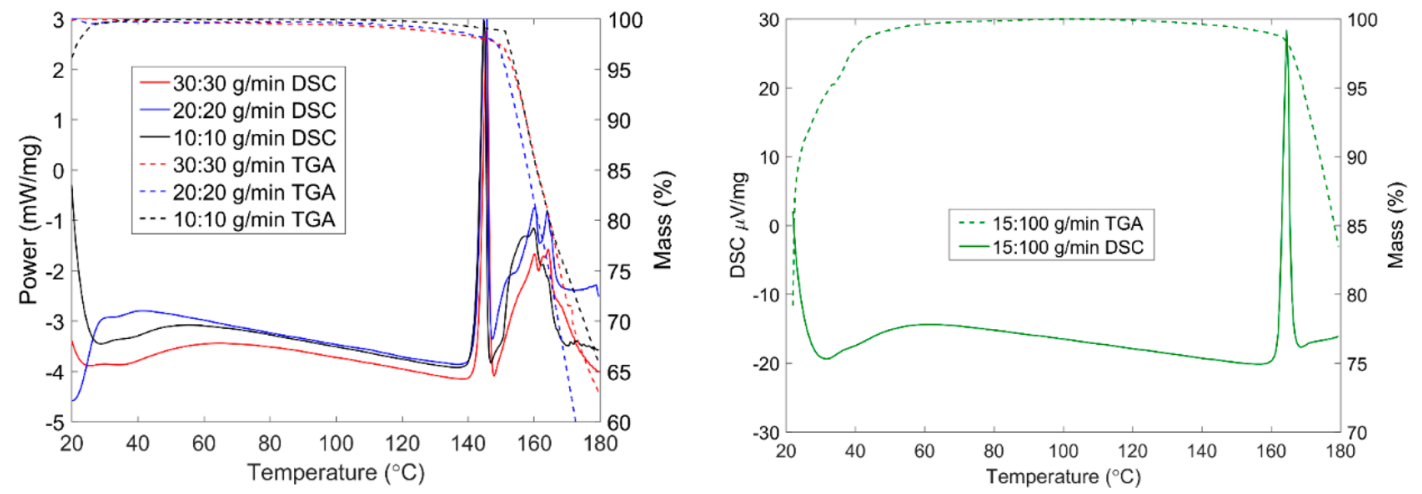

Figure 11. DSC/TGA for samples for 2:1 (left) and 1:1 (right) cocrystal from continuous runs. 


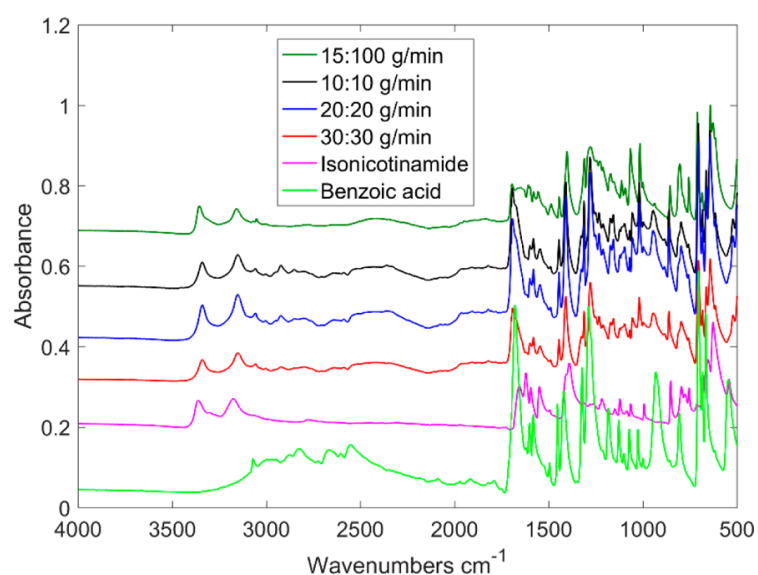

Figure 12. ATR-IR spectra for starting material and samples from continuous runs.

We showed that both 2:1 and 1:1 pure forms of the cocrystal can be produced using the combined approach method based on mixing-induced supersaturation in a continuous crystallization process. The combined antisolvent and reactive crystallization approach allows for additional flexibility in reaching specific phase regions in multicomponent systems using multiple solvents.

\section{CONCLUSIONS}

We have used Design of Experiments to find conditions suitable for formation of $2: 1$ and $1: 1$ cocrystals of benzoic acid and isonicotinamide by mixing ethanolic solutions of benzoic acid and aqueous solutions of isonicotinamide in isothermal conditions. The input parameters were the two solution concentrations and the mass ratio of the two solutions, while the output variables were the solid form produced, solid yield, induction time, and slurry flow rating. Optimal conditions were then identified for both 2:1 and 1:1 cocrystal formation, and continuous crystallization experiments were performed at several total flow rates. Both solid forms were selectively produced in continuous crystallization as confirmed by XPRD, IR, DSC, and NMR. Mixing conditions were found to influence the solid yield and particle size distribution of crystal produced although not the solid form.

The combined antisolvent and reactive crystallization approach can be a useful tool for reaching solid phases that are otherwise difficult to access through mixing-induced supersaturation. Under conditions of thermodynamic control, it may be possible to reach certain regions of phase diagrams that would be not accessible by other approaches due to nonideal shapes of some phase diagrams. Under conditions of kinetic control, it may be possible to influence nucleation kinetics through the initial mixing of two solutions, which would depend on compositions of both solutions as well as mixing conditions applied.

\section{ASSOCIATED CONTENT}

\section{S Supporting Information}

The Supporting Information is available free of charge on the ACS Publications website at DOI: 10.1021/acs.cgd.6b01866.

Detailed results of the DoE model, particle shape and size plots, example NMR spectra, XRPD results for continuous run carried out in the Ehrfeld MMRS (PDF)

\section{AUTHOR INFORMATION}

\section{Corresponding Author}

*E-mail: jan.sefcik@strath.ac.uk.

ORCID

Vaclav Svoboda: 0000-0002-2386-7112

Notes

The authors declare no competing financial interest.

\section{ACKNOWLEDGMENTS}

We would like to thank Maria Briuglia for assistance with Technobis Crystalline. The authors would like to thank EPSRC and the Doctoral Training Centre in Continuous Manufacturing and Crystallization (Grant ref: EP/K503289/1) for funding V.S. and J.M. The authors would like to thank EPSRC and Centre for Innovative Manufacturing in Continuous Manufacturing and Crystallization (Grant ref EP/I033459/1) for funding P.M. The authors would like to thank the International Strategic Partnership between University of Strathclyde and Nanyang Technological University Singapore for funding L.E.C. The authors would like to thank EPSRC for Fellowship (Grant ref EP/N015401/1) for funding I.D.H.O. The authors would like to acknowledge that this work was carried out in the CMAC National Facility supported by UKRPIF (UK Research Partnership Fund) award from the Higher Education Funding Council for England (HEFCE) (Grant ref HH13054).

\section{REFERENCES}

(1) Domingos, S.; André, V.; Quaresma, S.; Martins, I. C. B.; Minas da Piedade, M. F.; Duarte, M. T. J. Pharm. Pharmacol. 2015, 67, 830846.

(2) Elder, D. P.; Holm, R.; Diego, H. L. de. Int. J. Pharm. 2013, 453, 88-100.

(3) Trask, A. V. Mol. Pharmaceutics 2007, 4, 301-309.

(4) Friščić, T.; Jones, W. Cryst. Growth Des. 2009, 9, 1621-1637.

(5) Macfhionnghaile, P.; Hu, Y.; Gniado, K.; Curran, S.; Mcardle, P.; Erxleben, A. J. Pharm. Sci. 2014, 103, 1766-1778.

(6) Hasa, D.; Carlino, E.; Jones, W. Cryst. Growth Des. 2016, 16, $1772-1779$.

(7) Rehder, S.; Christensen, N. P. A.; Rantanen, J.; Rades, T.; Leopold, C. S. Eur. J. Pharm. Biopharm. 2013, 85, 1019-1030.

(8) Moradiya, H. G.; Islam, M. T.; Halsey, S.; Maniruzzaman, M.; Chowdhry, B. Z.; Snowden, M. J.; Douroumis, D. CrystEngComm 2014, 16, 3573-3583.

(9) Wittering, K.; King, J.; Thomas, L.; Wilson, C. Crystals 2014, 4, 123-140.

(10) Lee, M.-J.; Chun, N.-H.; Wang, I.-C.; Liu, J. J.; Jeong, M.-Y.; Choi, G. J. Cryst. Growth Des. 2013, 13, 2067-2074.

(11) Rodríguez-Hornedo, N.; Nehm, S. J.; Seefeldt, K. F.; PagánTorres, Y.; Falkiewicz, C. J. Mol. Pharmaceutics 2006, 3, 362-367.

(12) Zhao, L.; Raval, V.; Briggs, N. E. B.; Bhardwaj, R. M.; McGlone, T.; Oswald, I. D. H.; Florence, A. J. CrystEngComm 2014, 16, 57695780.

(13) Powell, K. A.; Bartolini, G.; Wittering, K. E.; Saleemi, A. N.; Wilson, C. C.; Rielly, C. D.; Nagy, Z. K. Cryst. Growth Des. 2015, 15, 4821-4836.

(14) Powell, K. A.; Croker, D. M.; Rielly, C. D.; Nagy, Z. K. Chem. Eng. Sci. 2016, 152, 95-108.

(15) Alvarez, A. J.; Myerson, A. S. Cryst. Growth Des. 2010, 10, 2219-2228.

(16) Brown, C. J.; Adelakun, J. A.; Ni, X. Chem. Eng. Process. 2015, 97, 180-186.

(17) Ferguson, S.; Morris, G.; Hao, H.; Barrett, M.; Glennon, B. Chem. Eng. Sci. 2013, 104, 44-54.

(18) Lindenberg, C.; Schöll, J.; Vicum, L.; Mazzotti, M.; Brozio, J. Chem. Eng. Sci. 2008, 63, 4135-4149. 
(19) Baxendale, I. R.; Braatz, R. D.; Hodnett, B. K.; Jensen, K. F.; Johnson, M. D.; Sharratt, P.; Sherlock, J.-P.; Florence, A. J. J. Pharm. Sci. 2015, 104, 781-791.

(20) Bałdyga, J.; Makowski, 1.; Orciuch, W. Chem. Eng. Res. Des. 2007, $85,745-752$.

(21) Leyssens, T.; Springuel, G.; Montis, R.; Candoni, N.; Veesler, S. Cryst. Growth Des. 2012, 12, 1520-1530.

(22) Munshi, T.; Redha, B.; Feeder, N.; Meenan, P.; Blagden, N. Cryst. Growth Des. 2016, 16, 1817-1823.

(23) Seaton, C. C.; Parkin, A.; Wilson, C. C.; Blagden, N. Cryst. Growth Des. 2008, 9, 47-56.

(24) Boyd, S.; Back, K.; Chadwick, K.; Davey, R. J.; Seaton, C. C. J. Pharm. Sci. 2010, 99, 3779-3786.

(25) Reus, M. A.; van der Heijden, A. E. D. M.; ter Horst, J. H. Org. Process Res. Dev. 2015, 19, 1004-1011.

(26) Redha, B. H. Thesis title: Impact of mixed solvent on co-crystal solubility, ternary diagrams and crystallisation scale-up. Crystallisations of Isonicotinamide-Benzoic Acid Co-crystals from Ethanol-Water Cosolvent System. University of Bradford, 2013.

(27) Al Nasser, W. N.; Pitt, K.; Hounslow, M. J.; Salman, A. D. Powder Technol. 2013, 238, 151-160.

(28) Aakeröy, C. B.; Beatty, A. M.; Helfrich, B. A. Angew. Chem., Int. Ed. 2001, 40, 3240-3242.

(29) Buanz, A.; Prior, T. J.; Burley, J. C.; Raimi-Abraham, B. T.; Telford, R.; Hart, M.; Seaton, C. C.; Davies, P. J.; Scowen, I. J.; Gaisford, S.; Williams, G. R. Cryst. Growth Des. 2015, 15, 3249-3256. 